\title{
CONFORMAL DISTORTION OF BOUNDARY SETS
}

\author{
D. H. HAMILTON
}

\begin{abstract}
Conformal maps $f$ of the disk into itself have the property that $\operatorname{dim} f^{-1}(F) \leq \operatorname{dim} F$ for any set $F$ on the unit circle.
\end{abstract}

1. Introduction. A common theme in complex analysis is the estimation of the Hausdorff dimension of special sets, for example the limit set of a Fuchsian group or the support of harmonic measure. A connecting link is the boundary distortion caused by conformal maps. For instance let $f$ map the unit disk $\mathbf{D}$ conformally onto a fundamental region $\Omega$ of a Fuchsian group. A classical lemma of Löwner [10] shows that the length of $\bar{\Omega} \cap \partial \mathbf{D}$ is greater than $f^{-1}(\bar{\Omega} \cap \partial \mathbf{D})$; however if the latter is zero this is of no use for estimating Hausdorff dimension. We prove

THEOREM 1. For any conformal map $f$ on $\mathbf{D}$, with $f(\mathbf{D}) \subset \mathbf{D}$ and set $E \subset \partial \mathbf{D}$ with angular limits $f(E) \subset \partial \mathrm{D}$ we have

$$
\operatorname{dim}(f(E)) \geq \operatorname{dim}(E) .
$$

The result is provided not by a distortion theorem for Hausdorff measures but from an estimate for $\alpha$-capacities. Recall that the inner $\alpha$-capacity $C_{\alpha}(E)$ of a set $E$ is defined by means of kernels

$$
k_{\alpha}(t)= \begin{cases}\log (1 / t), & \alpha=0 \\ 1 / t^{\alpha}, & 0<\alpha \leq 1\end{cases}
$$

so that

$$
C_{\alpha}(E)=k_{\alpha}^{-1}\left\{\inf _{\mu} \iint_{E E} k_{\alpha}(|x-y|) d \mu(x) d \mu(y)\right\},
$$

where the infimum is taken over all probability measures $\mu$ supported by compact subsets of $E$ (see Carleson [5]).

Now let $\mathscr{S}$ be the family of functions $f$ one-to-one analytic on $\mathbf{D}$, satisfying $f(0)=0$. Theorem 1 is an immediate consequence of

THEOREM 2. Suppose that for $f \in \mathscr{S}$ we have $f(\mathbf{D}) \subset \mathbf{D}$ and some $E \subset \partial \mathbf{D}$ with angular limits $f(E) \subset \partial \mathrm{D}$. Then for $0 \leq \alpha \leq 1$

$$
C_{\alpha}(f(E)) \geq\left|f^{\prime}(0)\right|^{-1 / 2} C_{\alpha}(E) .
$$

REMARKS. The case $\alpha=0$ is due to Pommerenke (see [18, p. 348]).

Actually our results are somewhat more general than the case $f(\mathbf{D}) \subset \mathbf{D}$ and $f(E) \subset \partial \mathbf{D}$. Suppose that for a general Riemann mapping $f: \mathbf{D} \rightarrow \Omega$ there is a

Received by the editors July 8, 1986.

1980 Mathematics Subject Classification (1985 Revision). Primary 30C35; Secondary 30C55, $30 \mathrm{C} 40$.

Research supported in part by NSF Grant 8501509 . 
Dinismooth curve $\gamma$ outside $\Omega$ so that $f(E) \subset \gamma$. By composition of conformal maps we deduce $\operatorname{dim}(f(E)) \geq \operatorname{dim}(E)$. This may be compared with a result of Markov [12] who proves for arbitrary $f$ and $E \subset \partial \mathbf{D} \operatorname{dim}(f(E)) \geq \psi(\operatorname{dim} E)$, where $\psi$ is an increasing function with $\psi(t) \geq 2 t /(1+t)$ as $t \rightarrow 1$. This sharpens a classical result of Beurling [3] that $\psi(t) \geq t / 2$. Beurling also proved that $C_{0}(f(E))=0$ implies $C_{0}(E)=0$. We extend Beurling's theory to general capacities.

DEFINITION 1. Let $h: \mathbf{R} \rightarrow \mathbf{R}$ be absolutely monotone, i.e., $h, h^{\prime}, h^{\prime \prime}, \ldots$ are positive. Define kernel $k(t)=h(\log (1 / t))$ and inner capacity

$$
C_{h}(E)=k^{-1}\left\{\inf \iint_{E E} k(|x-y|) d \mu(x) d \mu(y)\right\}
$$

where the infimum is taken over probability measures $\mu$ supported on compact subsets of $E$. We say that such a capacity is admissible if $\int_{0}^{1} h(\log (1 / t)) t d t<\infty$. Carleson [5] studied such capacities in the case that $h$ is monotone.

THEOREM 3. Suppose that the capacities $C_{j}(j=1,2)$ derived from kernels $h\left(\log \left(1 / t^{j}\right)\right)$ are admissible. Then for any function $f(z)=\sum_{n=0}^{\infty} a_{n} z^{-n}$ univalent in $\{|z|>1\}$ and for any $E \subset \partial \mathbf{D}$

$$
C_{1}(f(E)) \geq\left\{C_{2}(E)\right\}^{1 / 2} .
$$

COROLlARY 1. Suppose that $h$ is strongly monotone with polynomial growth. Then for any $f$ univalent on $\mathbf{D}$ and $E \subset \partial \mathbf{D}$ we have $C_{h}(E)=0$ whenever $C_{h}(f(E))=0$.

Beurling also studied the set $E \subset \partial \mathbf{D}$ on which a univalent function could be zero, showing that $C_{0}(E)=0$. On the other hand Carleson [4] shows that sets of positive logarithmic capacity need not be sets of uniqueness for the Dirichlet class. In [1] it was asked if $C_{0}(E)>0$ and $f_{1}=f_{2}$ on $E$ for $f_{j} \in \mathscr{S}$ implies $f_{1} \equiv f_{2}$. We show that there is a set $E \subset \partial \mathbf{D}$ of dimension 1 which is not a set of uniqueness for $\mathscr{S}$. Here there is a strong connection between fixed sets of $\mathscr{S}$ and zero sets of Hölder classes, (see also Döppel, Köditz and Timman [6]). In particular $E$ is the fixed set of an analytic flow if and only if $E$ is a Carleson set, i.e. $E$ has length zero and if its complementary subarcs have lengths $l_{n}$ satisfying $\sum l_{n} \log \left(1 / l_{n}\right)<\infty$.

2. Outline of the proof. The idea of the proof is easy to explain when we assume that the conformal map $f: \mathbf{D} \rightarrow \mathbf{D}$ has smooth extension to $\overline{\mathbf{D}}$.

First we use an inequality of Nehari (see §4) which holds for $f \in \mathscr{S}$ and $f(\mathbf{D}) \subset$ D. In the case that $f$ is smooth on $\partial \mathbf{D}$ and $E \subset \partial \mathbf{D}$ is mapped into $\partial \mathbf{D}$ the Nehari inequality may be transformed to

$$
\iint_{E E} \log \frac{1}{|f(x)-f(y)|} d \lambda(x) d \lambda(y) \leq \iint_{E E} \log \frac{1}{|x-y|} d \lambda(x) d \lambda(y)
$$

for all real measures $\lambda$ (supported on $E$ ) which are admissible, i.e.,

$$
\iint_{E E} \log \frac{1}{|x-y|} d \lambda(x) d \lambda(y)<\infty .
$$

Now as $E, f(E) \subseteq \partial \mathbf{D}$ the kernels are positive semidefinite so we may use a lemma of Schur (see §3) to "exponentiate" the inequalities. Thus we get for $0<\alpha \leq 1$

$$
\iint_{E E} \frac{d \lambda(x) d \lambda(y)}{|f(x)-f(y)|^{\alpha}} \leq \iint_{E E} \frac{d \lambda(x) d \lambda(y)}{|x-y|^{\alpha}}
$$


for all admissible measures $\lambda$, i.e.,

$$
\iint_{E E} \frac{d \lambda(x) d \lambda(y)}{|x-y|^{\alpha}}<\infty
$$

We then let $\lambda$ be a sequence of probability measures such that

$$
\iint_{E E} \frac{d \lambda(x) d \lambda(y)}{|x-y|^{\alpha}} \rightarrow\left\{C_{\alpha}(E)\right\}^{-\alpha}
$$

and thus from the definition of $\alpha$-capacity

$$
\left\{C_{\alpha}(f(E))^{-\alpha} \leq \inf \iint_{E E} \frac{d \lambda(x) d \lambda(y)}{|f(x)-f(y)|^{\alpha}} \leq\left\{C_{\alpha}(E)\right\}^{-\alpha} .\right.
$$

Unfortunately this does not work very well in general. We need to approximate $E$ by sets in $\mathbf{D}$ and then take care of the fact that our kernels are no longer positive semidefinite.

One might also expect to prove Theorem 1 from a result for Hausdorff measures. However it is not true that if $f \in \mathscr{S}$ maps $\mathbf{D}$ into $\mathbf{D}$ and $E \subset \partial \mathbf{D}$ into $\partial \mathbf{D}$ that $\Lambda(f(E)) \geq \Lambda(E)$ for any Hausdorff measure $\Lambda$. For instance there are $f$ mapping sets $E$ of positive logarithmic measure (but zero logarithmic capacity) to a single point. Consequently the method of Löwner's inequality will not work.

3. Quadratic inequalities. The method of exponentiating quadratic inequalties based on a lemma of Schur was introduced by Löwner [10] and FitzGerald [7]. Hamilton [9] introduced a continuous version for boundary value problems.

Let $A=\left(a_{i j}\right)_{n \times n}$ be $n \times n$ complex matrices. The previous theory depends on two notions of Schur for $A$ symmetric, $B$ hermitian positive definite

(i) Schur product $A B=\left(a_{i j} b_{i j}\right)_{n \times n}$,

(ii) Schur inequality $A<B$ :

$$
\left|\sum_{i=1}^{n} \sum_{j=1}^{n} a_{i j} \lambda_{i} \lambda_{j}\right| \leq \sum_{i=1}^{n} \sum_{j=1}^{n} b_{i j} \lambda_{i} \bar{\lambda}_{j}
$$

for all $\lambda_{1}, \ldots, \lambda_{n} \in \mathbf{C}$.

The fundamental result is $A<B \Rightarrow A^{2}<B^{2}$. In this paper we have the slightly different situation of real symmetric matrices and the inequality

$$
\sum_{i=1}^{n} \sum_{j=1}^{n} a_{i j} \lambda_{i} \lambda_{j} \leq \sum_{i=1}^{n} \sum_{j=1}^{n} b_{i j} \lambda_{i} \lambda_{j}
$$

for all real $\lambda_{1}, \ldots, \lambda_{n}$. Furthermore we know that $A$ and $B$ are positive semidefinite.

LEMMA 1. For all real $\lambda_{1}, \ldots, \lambda_{n}$ and $m=1,2, \ldots$

$$
\sum_{i=1}^{n} \sum_{j=1}^{n} a_{i j}^{m} \lambda_{i} \lambda_{j} \leq \sum_{i=1}^{n} \sum_{j=1}^{n} b_{i j}^{m} \lambda_{i} \lambda_{j}
$$

This is easily proved by induction noting that the Schur product is commutative: assuming $B^{m-1}-A^{m-1}>0$ and multiplying by $A>0$ Schur's lemma for products gives $A B^{m-1}-A^{m}>0$. On the other hand $B-A>0$ and multiplying by $B^{m-1}$ gives $B^{m}-B^{m-1} A>0$. Adding these two gives $B^{m}-A^{m}>0$, which proves the lemma. 
LEMMA 2. For any absolutely monotone function $h(t)$ :

$$
\sum_{i=1}^{n} \sum_{j=1}^{n} h\left(a_{i j}\right) \lambda_{i} \lambda_{j} \leq \sum_{i=1}^{n} \sum_{j=1}^{n} h\left(b_{i j}\right) \lambda_{i} \lambda_{j}
$$

for all $\lambda_{1}, \ldots, \lambda_{n} \in \mathbf{R}$.

This lemma follows by induction from Lemma 1 for all polynomials $h(t)$ with positive coefficients. Thus by approximation it holds for $h(t)=e^{\alpha t}, \alpha \geq 0$. Finally it is well known that for any absolutely monotone $h$

$$
h(t)=\int_{0}^{\infty} e^{\alpha t} d \mu(\alpha)
$$

for some positive measure $\mu$, which together with the above consideration proves (3).

Actually we need to consider continuous versions of (3).

LEMMA 3. Suppose that $A, B$ are real symmetric continuous on $\mathrm{D}^{2}$ and for any real-valued continuous function $\lambda(x)$ having compact support on $\mathbf{D}$ we have

$$
\begin{gathered}
\left\{\begin{array}{l}
\iint A(x, y) \lambda(x) \lambda(y)|d x|^{2}|d y|^{2} \geq 0 \\
\iint B(x, y) \lambda(x) \lambda(y)|d x|^{2}|d y|^{2} \geq 0
\end{array}\right. \\
\iint B(x, y) \lambda(x) \lambda(y)|d x|^{2}|d y|^{2} \geq \iint A(x, y) \lambda(x) \lambda(y)|d x|^{2}|d y|^{2} .
\end{gathered}
$$

Then for any absolutely monotone function $h(t)$

$$
\iint h(B(x, y)) \lambda(x) \lambda(y)|d x|^{2}|d y|^{2} \geq \iint h(A(x, y)) \lambda(x) \lambda(y)|d x|^{2}|d y|^{2}
$$

for all real-valued continuous $\lambda(x)$ with compact support.

This is proved from Lemma 2 by approximating with Riemann sums (see Hamilton [9]). Further approximation arguments show that we may allow $A, B$ to have singularities and $\lambda$ to be a suitable measure. Carleson [5] considers kernels with singularities of the form $k(t)=\log 1 / t$ and establishes potential theory for kernels $h(k)$. Carleson requires that $\int_{0}^{1} h(\log 1 / t) t d t<\infty$. Consequently we have a class of admissible real measures $\mu$ satisfying

$$
\iint_{\mathbf{D D}} h\left(\log \frac{1}{|x-y|}\right)|d \lambda(x)||d \lambda(y)|<\infty,
$$

i.e. the potential $\int h(\log (1 /|x-y|)) d \lambda(x)$ has finite energy. Putting these facts together we get

Proposition 1. Suppose that $A, B$ are real symmetric on $\mathbf{D}^{2}$, continuous except on the diagonal. Let

$$
|A(x, y)|+|B(x, y)| \leq C \log \frac{1}{|x-y|} .
$$

Then if (4), (5) are satisfied and $h$ is any absolutely monotone function such that $h(\log (1 /|z|)) \in L^{1}\left(\mathbf{R}^{2}\right)$ we have

$$
\iint h(B(x, y)) d \lambda(x) d \lambda(y) \geq \iint h(A(x, y)) d \lambda(x) d \lambda(y)
$$

for all real admissible measures supported on compact subsets of $\mathbf{D}$. 
4. Golusin and Nehari inequalities. The following two quadratic inequalities are essential.

THEOREM A (GOLUSIN [17]). Suppose that $f(z)=z+\sum_{n=1}^{\infty} a_{n} z^{-n}$ is univalent in $\Omega=\{|z|>1\}$. Then for any points $z \in \Omega(j=1, \ldots, n)$ and complex numbers $\lambda_{1}, \ldots, \lambda_{n}$

$$
\left|\sum_{i=1}^{n} \sum_{j=1}^{n} \lambda_{i} \lambda_{j} \log \right| \frac{f\left(z_{i}\right)-\left(z_{j}\right)}{z_{i}-z_{j}}|| \leq \sum_{i=1}^{n} \sum_{j=1}^{n} \lambda_{i} \bar{\lambda}_{j} \log \frac{1}{\left(1-1 / z_{i} \bar{z}_{j}\right)} .
$$

TheOREM B (Nehari [13]). Suppose that $f \in \mathscr{S}, f(\mathbf{D}) \subset \mathbf{D}$. Then

$$
0 \leq \sum_{i=1}^{n} \sum_{j=1}^{n} \lambda_{k} \lambda_{j} \log \left|\frac{f^{\prime}(0) z_{j} z_{k}}{f\left(z_{j}\right) f\left(z_{k}\right)} \frac{f\left(z_{j}\right)-f\left(z_{k}\right)}{z_{j}-z_{k}} \frac{1-f\left(z_{j}\right) \overline{f\left(z_{k}\right)}}{1-z_{j} \bar{z}_{k}}\right|
$$

for all $z_{n}, \ldots, z_{n} \in \mathbf{D}$ and $\lambda_{1}, \ldots, \lambda_{n} \in \mathbf{R}$.

The proof of continuous versions is immediate.

LEMMA 4. For $f \in \mathscr{S}, f(\mathbf{D}) \subset \mathbf{D}$

$$
\begin{aligned}
& \iint_{\mathbf{D D}} \log \frac{\left|f^{\prime}(0) x y\right|}{|(x-y)(1-x \bar{y})|} \lambda(x) \lambda(y)|d x|^{2}|d y|^{2} \\
& \quad \geq \iint_{\mathbf{D D}} \log \frac{|f(x) f(y)|}{\mid(f(x)-f(y))(1-f(x) \overline{f(y)} \mid} \lambda(x) \lambda(y)|d x|^{2}|d y|^{2}
\end{aligned}
$$

for all real continuous $\lambda$ supported on a compact subset of $\mathbf{D}$.

The quadratic inequality of Lemma B may be rearranged to give

$$
\begin{gathered}
\sum_{j \neq k} \sum_{j} \lambda_{j} \lambda_{k} \log \frac{\left|f\left(z_{j}\right) f\left(z_{k}\right)\right|}{\mid\left(f\left(z_{j}\right)-f\left(z_{k}\right)\right)\left(1-f\left(z_{j}\right) \overline{f\left(z_{k}\right)} \mid\right.} \\
\leq \sum_{j \neq k} \sum_{j} \lambda_{k} \log \frac{\left|f^{\prime}(0) z_{j} z_{k}\right|}{\left|\left(z_{j}-z_{k}\right)\left(1-z_{j} \overline{z_{k}}\right)\right|} \\
\quad+\sum_{j=1}^{n} \lambda_{j}^{2} \log \left|\frac{f^{\prime}\left(z_{j}\right)\left(1-\left|f\left(z_{j}\right)\right|^{2}\right)}{\left(1-\left|z_{j}\right|^{2}\right)}\right|,
\end{gathered}
$$

which on keeping $z_{j}$ in a fixed compact subset of $\mathbf{D}$ and using an approximation argument we can ensure that the diagonal sum tends to zero as $n \rightarrow \infty$. A similar argument yields

LEMMA 5. Suppose that $f(z)=z \sum_{n=1}^{\infty} a_{n} z^{-n}$ is univalent in $\Omega=\{|z|>1\}$. Then for any complex continuous $\lambda$ compactly supported on $\Omega$

$$
\begin{aligned}
& \left.\left|\iint_{\Omega \Omega} \lambda(x) \lambda(y) \log \frac{f(x)-f(y)}{x-y}\right| d x\right|^{2}|d y|^{2} \mid \\
& \quad \leq \iint_{\Omega \Omega} \lambda(x) \overline{\lambda(y)} \log \frac{1}{(1-1 / x \bar{y})}|d x|^{2}|d y|^{2} .
\end{aligned}
$$

Unfortunately the kernels of Lemma 5 are not (quite) positive semidefinite. Consequently we need to restrict our attention to a subset of $\mathbf{D}$ where the kernels are "nearly" positive semidefinite. 
5. Proof of Theorems 1 and 2. Without loss of generality we restrict our attention to compact sets $E \subset \partial \mathrm{D}$, with $C_{h}(E)>0$. In particular $C_{0}(E)>0$. For any $\varepsilon>0$ choose an open set $\widetilde{E}$ which is the union of Stolz angles with vertices on $E$ such that $\widetilde{E}$ and $f \widetilde{E}$ are subsets of the annulus $\{1-\varepsilon<|z|<1\}$ and there is a compact $F \subset \widetilde{E}$ with

$$
\left|C_{h}(E)-C_{h}(F)\right|<\delta
$$

and provided $C_{h}(f(\widetilde{E}))<\infty$,

$$
\left|C_{h}(f(E))-C_{h}(f(F))\right|<\delta .
$$

First we note

LEMMA 6. The kernel $\log (1 /|1-\bar{x} y|)$ is positive semidefinite on $\mathbf{D}$.

We write

$$
\int_{\mathbf{D}} \log \frac{1}{(1-\bar{x} y)} d \mu(y)=\sum_{n=1}^{\infty} \frac{\mu_{n}}{n} \bar{x}^{n},
$$

where $\mu_{n}=\int y^{n} d \mu(y)$. Hence as $\mu$ is real

$$
\iint_{\mathbf{D D}} \log \frac{1}{(1-\bar{x} y)} d \mu(x) d \mu(y)=\sum_{n=1}^{\infty} \frac{\left|\mu_{n}\right|^{2}}{n}
$$

for which we take real parts to get the required result.

Also we prove

LEMMA 7. The kernel $\log |(1-x \bar{y}) /(x-y)|$ is positive semidefinite on $\mathbf{D}$.

Let us define $G(x, y)=\log |(1-\bar{x} y) /(x-y)|$, i.e., the Green's function of $\mathbf{D}$. Thus by Riesz representation of harmonic functions (see [5]) if we define

$$
u(x)=\int_{\mathbf{D}} G(x, y) d \mu(y),
$$

provided $d \mu=\mu(y)|d y|^{2}$ where $\mu$ is $C^{2}$ then $\Delta u=(-2 \pi) \mu$ and $u=0$ on $\partial \mathrm{D}$. Consequently from Green's formulae

$$
\begin{aligned}
\iint_{\mathbf{D D}} & \log \left|\frac{1-x \bar{y}}{x-y}\right| d \mu(x) d \mu(y) \\
= & (-2 \pi) \int_{\mathbf{D}} u \Delta u|d x|^{2} \\
= & (2 \pi) \int_{\mathbf{D}}|\nabla u|^{2}|d x|^{2}>0
\end{aligned}
$$

which proves the lemma.

Thus combining these results

LEMMA 8. The kernel $\log (1 /|(x-y)(1-\bar{x} y)|)$ is positive semidefinite on $\mathbf{D}$.

This follows from writing the kernel as

$$
2 \log \frac{1}{|1-\bar{x} y|}+\log \left|\frac{1-x \bar{y}}{x-y}\right|
$$

which are positive kernels by the previous lemma. 
Finally we deal with the $\log |x y|$ terms. We restrict our attention to a thin annulus $A:\{1-\delta \leq|x|,|y| \leq 1\}$. Now (even on $A$ ) $\log |x y|$ has positive and negative eigenvalues, so we find a kernel $K(x, y, \varepsilon)$ on $A$ such that $\log |x y|+K(x, y, \varepsilon)$ and $\log |f(x) f(y)|+K(x, y, \varepsilon)$ are positive semidefinite on $A$; also we require that

$$
\lim _{\varepsilon \rightarrow 0} \sup _{A}|K(x, y, \varepsilon)|=0 .
$$

To do this we use spectral resolution of the real symmetric kernel $\log |x y|$. On $L^{2}(A)$ the operator $\mathscr{L} g=\int_{A}\{\log |x y|\} g(y)|d y|^{2}$ spans a space with basis $1, \log |x|$. Thus $\mathscr{L}$ has rank 2 and

$$
\log |x y|=\lambda_{1} \phi_{1}(x) \phi_{1}(y)+\lambda_{2} \phi_{2}(x) \phi_{2}(y)
$$

where $\lambda_{j}, \phi_{j}$ are eigenvalues and orthonormal eigenfunctions of $\mathscr{L}$. Suppose that $\lambda_{1}>0$ and $\lambda_{2}<0$. Then we set

$$
K(x, y, \varepsilon)=-\lambda_{2} \phi_{2}(x) \phi_{2}(y)
$$

and we shall see later that it has the required properties. First we need to estimate the $\lambda_{j}$. It is clear that we need only consider the effect of $\mathscr{L}$ on functions of the form

$$
g(y)=\alpha+\beta \log |y|
$$

Then

$$
\mathscr{L} g=\left(\tau_{1} \alpha+\tau_{2} \beta\right)+\left(\tau_{0} \alpha+\tau_{1} \beta\right) \log |x|
$$

where

$$
\tau_{0}=\int_{A} 1 \cdot|d y|^{2}, \quad \tau_{1}=\int_{A} \log |y||d y|^{2}
$$

and

$$
\tau_{2}=\int_{A}(\log |y|)^{2}|d y|^{2} .
$$

Consequently the equation for eigenvalues is

$$
\left[\begin{array}{ll}
\tau_{1} & \tau_{2} \\
\tau_{0} & \tau_{1}
\end{array}\right]\left[\begin{array}{l}
\alpha \\
\beta
\end{array}\right]=\lambda\left[\begin{array}{l}
\alpha \\
\beta
\end{array}\right] .
$$

First we estimate the $\tau_{j}$ to $O\left(\varepsilon^{5}\right)$; a simple computation yields

LEMMA 9. Under the above notation

$$
\begin{aligned}
& \tau_{0}=2 \pi\left(\varepsilon-\frac{1}{2} \varepsilon^{2}\right) \\
& \tau_{1}=2 \pi\left\{-\frac{\varepsilon^{2}}{2}+\frac{\varepsilon^{2}}{6}+\frac{\varepsilon^{4}}{24}+O\left(\varepsilon^{5}\right)\right\} \\
& \tau_{2}=2 \pi \frac{\varepsilon^{3}}{3}+O\left(\varepsilon^{5}\right) .
\end{aligned}
$$

A direct computation now yields the eigenvalues $(j=1,2)$

$$
\begin{aligned}
\lambda_{j} & =\tau_{1}+(-1)^{j} \sqrt{\tau_{0} \tau_{2}} \\
& =2 \pi\left\{\left(-\frac{1}{2}+\frac{(-1)^{j}}{\sqrt{3}}\right) \varepsilon^{2}+\left(\frac{1}{6}+\left(-\frac{(-1)^{j}}{4 \sqrt{3}}\right)\right) \varepsilon^{2}+\cdots\right\} .
\end{aligned}
$$


Next we compute the eigenfunctions

$$
\psi_{j}=\alpha_{j}+\beta_{j} \log |x|
$$

For $j=1$ we solve

$$
\left[\begin{array}{cc}
\left(\frac{1}{\sqrt{3}} \varepsilon^{2}+\cdots\right) & \frac{\varepsilon^{3}}{3}+\cdots \\
\varepsilon- & \left(\frac{1}{\sqrt{3}} \varepsilon^{2}+\cdots\right)
\end{array}\right]\left[\begin{array}{l}
\alpha \\
\beta
\end{array}\right]=\left[\begin{array}{l}
0 \\
0
\end{array}\right] .
$$

Thus we can solve to get

$$
\psi_{1}=(-\sqrt{3} \varepsilon)+\log |y|
$$

Then

$$
\begin{aligned}
\int_{A}\left|\psi_{1}\right|^{2}|d y|^{2} & =3 \varepsilon^{2} \tau_{0}-2 \sqrt{3} \varepsilon \tau_{1}+\tau_{2} \\
& =2 \pi \varepsilon^{3}\left(3+\sqrt{3}+\frac{1}{3}+\cdots\right) .
\end{aligned}
$$

Consequently the normalized eigenfunction is

$$
\phi_{1}=\frac{-\varepsilon^{-1 / 2}}{\sqrt{2 \pi\left(1+1 / \sqrt{3}+\frac{1}{9}\right)}}+\frac{-\varepsilon^{-3 / 2} \log |y|}{\sqrt{2 \pi\left(3+\sqrt{3}+\frac{1}{3}\right)}} .
$$

In particular we have, for $x, y \in A, \lambda_{1} \phi_{1}(x) \phi_{1}(y)=O\left(\varepsilon^{2} \varepsilon^{-1}\right)=O(\varepsilon)$ as $\log |x|=$ $O(\varepsilon)$ on $A$. We then obtain a similar result for the other eigenvalue. Let us summarize this in

LEMMA 10. As $\varepsilon \rightarrow 0 ; x, y \in\{1-\varepsilon<|z|<1\}$.

$$
\lambda_{j} \phi_{j}(x) \phi_{j}(y)=O(\varepsilon) \text {. }
$$

In particular we see that the function $K(x, y, \varepsilon)$ is $O(\varepsilon)$. $1\}$ :

LEMMA 11. The following kernels are positive semidefinite on $\{1-\varepsilon<|x|,|y|<$

$$
K_{1}=\log |x y|+K(x, y, \varepsilon), \quad K_{2}=K(x, y, \varepsilon) .
$$

This is because, by explicit construction, the eigenvalue of $K_{j}$ is $(-1)^{j+1} \lambda_{j}$. Next we consider the kernel $\log |f(x) f(y)|$. It would be most complicated to follow through the above procedure; however by change of variables we can easily reduce to the above case. Also we need to observe that we are restricting ourselves to $x, y \in \widetilde{E}, f(\widetilde{E}) \subset\{1-\varepsilon<|z|<1\}$.

LEMMA 12. The following kernels are positive semidefinite on $\widetilde{E}$ :

$$
\begin{aligned}
& K_{3}=\log |f(x) f(y)|+K(f(x), f(y), \varepsilon), \\
& K_{4}=K(f(x), f(y), \varepsilon) .
\end{aligned}
$$

To prove this we observe that

$$
K_{3}=K_{1}(f(x), f(y))=\lambda_{1} \phi_{1}(f(x)) \phi_{1}(f(y))
$$

and

$$
K_{4}=\lambda_{2} \phi_{2}(f(x)) \phi_{2}(f(y))
$$

and each of these is positive semidefinite.

REMARKS. If $K_{1}, K_{2}$ had contained more than one eigenfunction the above substitution would not have worked. Finally we have, analogous to Lemma 10, 
LEMMA 13. As $\varepsilon \rightarrow 0, x, y \in \widetilde{E} K_{j}(x, y)=O(\varepsilon)$.

We are now ready to prove Theorem 1 . We add

$$
\iint\left(K_{2}(x, y)+K_{4}(x, y)\right) \lambda(x) \lambda(y)|d x|^{2}|d y|^{2}
$$

to both sides of the quadratic inequality of Lemma 4 , to obtain for admissible $\lambda$ :

$$
\begin{aligned}
\iint_{\widetilde{E} \widetilde{E}} A(x, y) \lambda(x) \lambda(y)|d x|^{2}|d y|^{2} \\
\leq \iint_{\widetilde{E} \widetilde{E}}\left(B(x, y)+\log \left|f^{\prime}(0)\right|\right) \lambda(x) \lambda(y)|d x|^{2}|d y|^{2} \\
\leq \iint_{\widetilde{E} \widetilde{E}} B(x, y) \lambda(x) \lambda(y)|d x|^{2}|d y|^{2}
\end{aligned}
$$

as $\left|f^{\prime}(0)\right| \leq 1$. Now

$$
A(x, y)=\log \frac{|f(x) f(y)|}{|(f(x)-f(y))(1-f(x) \overline{f(y)})|}+K_{2}+K_{4}
$$

and

$$
B(x, y)=\log \frac{|x y|}{|(x-y)(1-\bar{x} y)|}+K_{2}+K_{4} .
$$

By our previous lemmas, $A$ and $B$ are positive semidefinite. Thus we may apply Proposition 1, i.e., for any absolutely monotone $h$ with $h(\log (1 /|z|)) \in L^{1}\left(\mathbf{R}^{2}\right)$, letting $h_{0}(t)=h(t / 2)$

$$
\iint h_{0}(A(x, y)) d \lambda(x) d \lambda(y) \leq \iint h_{0}(B(x, y)) d \lambda(x) d \lambda(y)
$$

for all admissible real measures $d \lambda$. Next we note that for any $\delta>0$, there is an $\varepsilon>0$ and an $\widetilde{E}$ such that

$$
\begin{gathered}
h(A(x, y))=h_{0}\left(\log \frac{1}{|f(x)-f(y)|^{2}}\right)+O(\delta), \\
h(B(x, y))=h_{0}\left(\log \frac{1}{|x-y|^{2}}\right)+O(\delta)
\end{gathered}
$$

uniformly on $\widetilde{E}$. This follows from Lemma 13 and the definition of $\widetilde{E}$. Consequently we have

$$
\begin{array}{r}
\iint_{\widetilde{E} \widetilde{E}} h\left(\log \frac{1}{|f(x)-f(y)|}\right) d \lambda(x) d \lambda(y) \\
\quad \leq \iint_{\widetilde{E} \widetilde{E}} h\left(\log \frac{1}{|x-y|}\right) d \lambda(x) d \lambda(y)+O(\delta)
\end{array}
$$

for any measure $\mu$ with

$$
\iint_{\widetilde{E} \widetilde{E}} h\left(\log \frac{1}{|x-y|}\right) d \lambda(x) d \lambda(y)
$$

finite. We now let our measures be probability measures supported by $F$. In particular let $\lambda_{n}$ be a sequence such that

$$
\iint_{\widetilde{F} \widetilde{F}} h\left(\log \frac{1}{|x-y|}\right) d \lambda_{n}(x) d \lambda_{n}(y)
$$


tends toward the infimum. Thus by the above inequality

$$
\begin{aligned}
\inf _{\lambda} \iint_{f(F) f(F)} & h\left(\log \frac{1}{|x-y|}\right) d \lambda(x) d \lambda(y) \\
& \leq \inf _{\lambda} \iint_{F F} h\left(\log \frac{1}{|x-y|}\right) d \lambda(x) d \lambda(y)+\delta .
\end{aligned}
$$

Consequently recalling the construction of $F$, letting $\delta \rightarrow 0$ and taking $e^{h^{-1}}$ of both sides gives us a generalization of Theorem 2:

Proposition 2. Suppose that $f \in \mathscr{S}, f(\mathbf{D}) \subset \mathbf{D}$ and $E \subset \partial \mathbf{D}$ with $f(E) \subset$ $\partial \mathrm{D}$. Then for any admissible capacity

$$
C_{h}(f(E)) \geq C_{h}(E) .
$$

This is not quite a generalization of Theorem 1 because we lost our $\left|f^{\prime}(0)\right|$ factor. To prove Theorem 1 we begin with

$$
\iint_{\widetilde{E} \widetilde{E}} A_{1}(x, y) d \lambda(x) d \lambda(y) \leq \iint_{\widetilde{E} \widetilde{E}} B(x, y) d \lambda(x) d \lambda(y)
$$

where $A_{1}=A(x, y)+\log \left(1 /\left|f^{\prime}(0)\right|\right)$ which is positive semidefinite. We then follow through the previous method with explicit function $h(t)=e^{\alpha t}$ and pick up the extra factor.

Theorem 1 follows immediately from Frostman's relation between capacity and Hausdorff measure (see [5]).

6. Proof of Theorem 3. The argument here is similar to the previous section so we just sketch it. We use the continuous form of the Golusin inequalty to write

$$
\begin{aligned}
\left.\left|\iint \lambda(x) \lambda(y) \log \frac{1}{|f(x)-f(y)|}\right| d x\right|^{2}|d y|^{2} \mid \\
\leq \iint \lambda(x) \lambda(y) \log \frac{1}{|1-1 / x \bar{y}|}|d x|^{2}|d y|^{2} \\
\quad+\left.\left|\iint \lambda(x) \lambda(y) \log \frac{1}{|x-y|}\right| d x\right|^{2}|d y|^{2} \mid
\end{aligned}
$$

for all real-valued continuous $\lambda$ compactly supported on $\Omega$. Then as in $\S 2$ we restrict ourselves to $\lambda$ supported on $\{1<|z|<1+\varepsilon\}$.

From an approximation argument similar to that of $\S 2$

$$
\log \frac{1}{|x-y|}=\log \frac{1}{|1-1 / x \bar{y}|}+A(x, y, \varepsilon),
$$

where $A(x, y, \varepsilon) \rightarrow 0$ uniformly as $\varepsilon \rightarrow 0$. Thus we get

$$
\begin{aligned}
\left.\left|\iint \lambda(x) \lambda(y) \log \frac{1}{|f(x)-f(y)|}\right| d x\right|^{2}|d y|^{2} \mid \\
\leq 2 \iint \lambda(x) \lambda(y) \log \frac{1}{|1-1 / x \bar{y}|}|d x|^{2}|d y|^{2} \\
+\left.\left|\iint \lambda(x) \lambda(y) A(x, y, \varepsilon)\right| d x\right|^{2}|d y|^{2} \mid
\end{aligned}
$$


where $A(x, y, \varepsilon)$ uniformly tends to 0 as $\varepsilon \rightarrow 0$, and is positive semidefinite. Now any such quadratic inequality for real kernels implies the same inequality with complex $\lambda(x)$ supported in $\{1<|z|<1+\varepsilon\}$, see FitzGerald and Horn [8]. Consequently for any complex-valued continuous $\lambda(x)$ compactly supported on $\{1<|z|<1+\varepsilon\}$ :

$$
\begin{aligned}
& \left.\left|\iint \lambda(x) \lambda(y) \log \frac{1}{|f(x)-f(y)|}\right| d x\right|^{2}|d y|^{2} \mid \\
& \quad \leq \iint \lambda(x) \bar{\lambda}(y)\left\{\log \frac{1}{|1-1 / x \bar{y}|^{2}}+A(x, y)\right\}|d x|^{2}|d y|^{2} .
\end{aligned}
$$

We may then "exponentiate" this expression in accordance with Schur's lemma. Thus for any absolutely monotone function $h$

$$
\begin{aligned}
& \left.\left|\iint \lambda(x) \lambda(y) h\left\{\log \frac{1}{|f(x)-f(y)|}\right\}\right| d x\right|^{2}|d y|^{2} \mid \\
& \leq \iint \lambda(x) \lambda(y) h\left\{\log \frac{1}{|1-1 / x \bar{y}|^{2}}\right\}|d x|^{2}|d y|^{2}+O(\varepsilon),
\end{aligned}
$$

for all continuous positive $\lambda(x)$ supported in $\{1<|z|<1+\varepsilon\}$, and satisfying $\int \lambda(x)|d x|^{2}=1$. To complete the proof of Theorem 2 we take sets $\widetilde{E} \subset\{1<|z|<$ $1+\varepsilon\}$ such that $C_{h}(E)$ is approximated by $C_{h}(\widetilde{E})$, and $C_{h}(f(E))$ by $C_{h}(f(\widetilde{E}))$. Thus the proof in this section is just a slight variation of that in the previous section.

Finally we prove the Corollary. Notice that if $h$ has slow growth then for any set $E$ :

$$
C_{2}(E) \geq C_{1}(E)^{n} .
$$

To see this one simply inspects the kernels. Consequently by Theorem $3 C_{h}(f(E))=$ 0 implies $C_{h}(E)=0$.

7. Fixed points of conformal maps. Carleson sets are exactly the zero sets of the class $A_{\alpha}$ of functions $h(z)$ analytic on $\mathbf{D}$ and Hölder continuous with exponent $\alpha, 0<\alpha \leq 1$, on $\overline{\mathbf{D}}$, see [4].

Now we define the concept of a holomorphic conformal flow. We say that a class of functions $f_{\lambda} \in \mathscr{S}$, with parameter $\{|\lambda|<1\}$ is a holomorphic flow if

(i) $f_{\lambda}(z)$ is holomorphic in $\lambda \in \mathbf{D}$ for fixed $z$.

(ii) $f_{0}(z) \equiv z$.

The importance of holomorphic flows comes from quasiconformal theory. A set $E \subset \partial \mathrm{D}$ is a fixed set for a holomorphic flow $f_{\lambda}$ if $f_{\lambda}(z)=z$ for $z \in E,|\lambda|<1$.

PROPOSITION 3. A set $E \subset \partial \mathrm{D}$ of positive capacity is a fixed set for a holomorphic conformal flow if and only if $E$ is a Carleson set.

Now if $E$ is a Carleson set there is a nontrivial $h \in A_{1}$ with $h(0)=0,\left\|h^{\prime}\right\|_{\infty}<1$ and $h(z)=0$ on $E$. Then we set

$$
f_{\lambda}(z)=z+\lambda h(z)
$$

and note that for $|\lambda|<1, z_{j} \in \mathbf{D}$

$$
\left|f_{\lambda}\left(z_{1}\right)-f_{\lambda}\left(z_{2}\right)\right| \geq\left|z_{1}-z_{2}\right|\left(1-|\lambda|\left\|h^{\prime}\right\|_{\infty}\right),
$$

which implies that $f_{\lambda} \in \mathscr{P}$. 
Conversely, if $f_{\lambda}$ is a holomorphic conformal flow then by the $\lambda$-lemma of Bers and Royden [2], $f_{\lambda}(z)$ has a quasiconformal extension to $\mathbf{C}$ for $|\lambda|<1$. Consequently, $E$ is a zero set of the Hölder continuous function $f_{\lambda}(z)-z$. Therefore $E$ is a Carleson set.

The theorem shows that any Carleson set is a set of nonuniqueness for $\mathscr{S}$. We now display a Carleson set of dimension 1. It is easier to think of $\partial \mathbf{D}$ with normalized arc length as the interval $(0,1)$. We construct a Cantor set. At the first stage, remove an open interval $I_{1}$ of length $\left(1-\alpha_{1}\right)$, etc., so that at the $n$th stage we get a set $E_{n}$ consisting of $2^{n}$ subarcs of length $\alpha_{1} \cdots \alpha_{n-1}\left(1-\alpha_{n}\right) / 2^{n}$. To see that $E$ is a Carleson set, note $|E| \leq \alpha_{1} \cdots \alpha_{n}$ and

$$
\sum_{j} l_{j, n} \log \left(l_{j, n}^{-1}\right)=\sum_{n=1}^{\infty}\left(\alpha_{1} \cdots \alpha_{n-1}\right)\left(1-\alpha_{n}\right) \log \frac{2^{n}}{\left(\alpha_{1} \cdots \alpha_{n-1}\right)\left(1-\alpha_{n}\right)} .
$$

Let us choose $\alpha_{n}=1-n^{-1 / 2}$. Then as $n \rightarrow \infty$

$$
\alpha_{1} \cdots \alpha_{n} \rightarrow 0
$$

while

$$
\sum_{j} l_{j, n} \log \left(l_{j, n}^{-1}\right) \leq C_{1} \sum(n-1) e^{c_{2}-n^{1 / 2}}<\infty .
$$

On the other hand $E$ has dimension 1 since if the $\alpha_{j}$ are constant $\alpha<1, \operatorname{dim} E=$ $\log 2 / \log \frac{2}{\alpha}$. However the Hausdorff dimension of $E$ is not affected by finitely many $\alpha_{j}$. Thus as $\alpha_{n} \rightarrow 1$ we get $\operatorname{dim} E=1$.

If we have $f \in \mathscr{S}$, with $f(\mathbf{D}) \subset \mathbf{D}$ then Theorem 1 says that any fixed set must have zero capacity. We now use the Löwner differential equation to generate fixed sets $E \subset \partial \mathbf{D}$ of flows $f_{t} \in \mathscr{S}, 0 \leq t \leq 1$, and $f_{t}(\mathbf{D}) \subset \mathbf{D}$.

PROPOSITION 4. Let $p(z)$ be analytic on $\mathbf{D}$, and

(i) $p(z)=0, z \in E$,

(ii) $p(0)=1, \operatorname{Re} p>0$ on $\mathbf{D}$,

(iii) $p(z)$ has modulus of continuity $\omega(t)$ on $\overline{\mathbf{D}}$ satisfying

$$
\int_{0}^{1} \frac{d t}{\omega(t)}=\infty
$$

Then the solution of the system $\dot{f}_{t}=-f_{t} p\left(f_{t}\right), f_{0}(z)=z$ is univalent on $\mathbf{D}$, continuously differentiable with respect to $t$ on $\overline{\mathbf{D}}$, and satisfies $f_{t}(z)=z$ for $z \in E$.

We do not include the complete details of the proof since it is only a variation of the standard one for the Löwner equation, see [18]. The existence and uniqueness of solutions of first-order systems can be proved under assumption (iii), the so-called Osgood condition. The uniqueness of solutions implies $f_{t}$ is one-to-one on $\overline{\mathbf{D}}$.

Finally we observe that Propositions 3 and 4 actually have an application to "peak sets" of Hölder classes. Recall that $E \subset \partial \mathrm{D}$ is a peak set of a function $h$ analytic on $\mathbf{D}$, continuous on $\overline{\mathbf{D}}$, if $\operatorname{Re} h>0$ on $\mathbf{D}$ but $h(z)=0$ on $E$. B. A. Taylor and D. Williams [18] prove that the peak set of the Lipschitz class $A_{1}$ is a finite set, while Noell and Wolff [15] prove that every peak set of $A_{\alpha}$ has $(1-\alpha)$ Hausdorff measure zero. These peak sets are closely related to the fixed points of Proposition 4. Observe that $E$ is a peak set of $p$ implies $E$ is a fixed set for $f_{t}$. We define a 
general class of functions $A_{\omega}$ as follows. $A_{\omega}$ consists of functions $h$ analytic on D with modulus of continuity $w(t)$. Now if $\int_{0}^{1} d t / \omega(t)=\infty$ then $A_{\omega}$ falls between $A_{1}$ and $\bigcap_{\alpha<1} A_{\alpha}$. Thus by Proposition 4 any peak set of $A_{\omega}$ is a fixed set for $S$, with $f(D) \subset D$. Consequently from Theorem 1 we deduce 0 .

COROLlary 2. Let $E$ be a peak set of $A_{\omega}$, where $\int_{0}^{1} d t / \omega=\infty$. Then $C_{0}(E)=$

\section{REFERENCES}

1. K. Barth, D. Brannan and W. K. Hayman, Research problems in complex analysis, Bull. London Math. Soc. 16 (1984), 490-517.

2. L. Bers and H. Royden, Holomorphic families of injections, Acta Math. 157 (1986), 259-286.

3. A. Beurling, Ensembles exceptionelles, Acta Math. 72 (1940), 1-13.

4. L. Carleson, Sets of uniqueness for functions analytic in the unit disk, Acta Math. 87 (1952), 325-345.

5. _ Selected problems on exceptional sets, Van Nostrand, 1967.

6. K. Döppell, H. Köditz and S. Timman, Bemurkungen über Fixpunktmengen schlichter Functionen, Rend. Inst. Math. Univ. Trieste 8 (1976), 162-166 (1977).

7. C. H. FitzGerald, Quadratic inequalities and coefficient estimates for schlicht functions, Arch. Rational Mech. Anal. 46, 356-368.

8. C. H. FitzGerald and R. Horn, On the structure of hermitian symmetric matrices, J. London Math. Soc. (2) 15 (1977), 419-430.

9. D. H. Hamilton, Quadratic inequalities and interpolation, J. Analyse Math. 43 (1984), 26-50.

10. C. Löwner, Untersuchungen über schlichte konforme Abbildungen des Einheitskreises, Math. Ann. 89, 103-121.

11. N. G. Makarov, On the distortion of boundary sets under conformal mappings, Proc. London Math. Soc. (3) 51 (1985), 369-384.

12. Conformal mapping and Hausdorff measures, (preprint).

13. Z. Nehari, Some inequalities in the theory of functions, Trans. Amer. Math. Soc. 75 (1953), 256-286.

14. R. Nevanlinna, Analytic functions, Springer-Verlag, 1970.

15. A. V. Noell and T. H. Wolff, On peak sets for Lip $\alpha$ classes, J. Functional Anal. (to appear).

16. C. Pommerenke, On the logarithmic capacity and conformal mapping, Duke Math. J. 35 (1968), 321-326.

17. __ Univalent functions, Vandenhoeck and Ruprecht, Göttingen, 1975.

18. B. H. Taylor and D. L. Williams, The peak sets of $A^{m}$, Proc. Amer. Math. Soc. 24 (1970), 604-606.

Department of Mathematics, University of Maryland, College Park, MaryLAND 20742 